\title{
A finite element modeling of thermal conductivity of fabrics embedded with phase change material
}

\author{
Y. Ding ${ }^{1} \quad$ J. A. Gear ${ }^{2} \quad$ K. N. Tran ${ }^{3}$
}

(Received 13 August 2007; revised 24 December 2007)

\begin{abstract}
The thermal capacitance of a fabric is increased when the fabric is embedded with a phase change material ( $\mathrm{PCM})$. This is due to utilizing the latent heat release or absorption of the PCM during its phase change process. This article presents a modified finite element method algorithm based on the Galerkin weak formulation with quadratic shape functions. The modification correctly models the phase change process. A diver's dry suit made from four types of garments, 1-layer Thinsulate, 1-layer PCM, and 2-layer and 4-layer Thinsulate-PCM composites, are investigated using the modified finite element method. Temperature profiles and heat fluxes are compared with and are shown to be superior to results obtained using a finite difference procedure.
\end{abstract}

See http://anziamj.austms.org.au/ojs/index.php/ANZIAMJ/article/view/379 for this article, (c) Austral. Mathematical Soc. 2008. Published February 27, 2008. ISSN $1446-8735$ 


\section{Contents}

1 Introduction

C440

2 Governing equations

C441

3 The Galerkin finite element discretization

C444

4 Results and discussions

C448

5 Conclusions

C453

References

C454

\section{Introduction}

A Phase Change Material (PCM) is a type of polymer substance. It performs like a conventional thermal energy storage in its solid or liquid phase, that is, its temperature rises as it absorbs heat and vice versa. However, when a PCM reaches the temperature at which it changes phase (its melting point if from solid to liquid phase, or fusion temperature if from liquid to solid phase) it absorbs or releases large amounts of heat without a significant rise or drop in temperature [6]. Compared to conventional thermal storage materials such as rock and water, PCMs generally have the capacity to store about 3 to 14 times more heat per unit volume within the human comfort range of 20 to $30^{\circ} \mathrm{C}$.

One of the applications of PCMs is in the clothing industry. For example, PCM may be used in specialized military clothing, athletic clothing or protective clothing for fire-fighters $[1,4,5,8,7]$. In this application, the PCMs are encapsulated in either microscopic or macroscopic balls of heat resistant plastic. When embedded into a fabric, they increase the thermal capacitance of the fabric by utilizing the latent heat release or absorption during the 
phase change process to keep a wearer warmer or cooler over an extended period.

For the design of new enhanced garments, it is necessary to model the thermal performance of PCM embedded fabrics. Considering the size ratio of a garment surface to its thickness is roughly 100:1, Gear et al. [2] modeled the phase change process as a one dimensional heat flow in a continuum using a finite difference scheme. Both Nuckols [8] and Gear et al. [2] modeled a diver's dry suit made of a 2-layer fabric composed from a foam enhanced with microencapsulated PCM C18 paraffin (octadecane) in Thermosorb ${ }^{1}$ and a microfibrous insulating material called Thinsulate ${ }^{2}$. The temperature profiles and the final phase change positions predicted by the numerical results of Gear et al. [2] agreed well with the results of Nuckols.

Using the same mathematical model as in Gear et al. [2], this article presents a modified finite element method (FEM) based on the Galerkin weighted residual weak formulation with quadratic shape functions. The modification correctly models the phase change process. The implementation of the FEM procedure is used to solve for the transient heat transfer through the thickness of a diver dry suit made from the same fabrics as those used by Nuckols [8] and Gear et al. [2].

\section{Governing equations}

The one dimensional heat equation given by Hill and Dewynn [3] is

$$
\frac{\partial h}{\partial t}=\frac{\partial}{\partial x}\left(k(u) \frac{\partial u}{\partial x}\right)
$$

where $u=u(x, t)$ is the temperature $\left[{ }^{\circ} \mathrm{C}\right], h(u)$ is the enthalpy $\left[\mathrm{J} \mathrm{m}^{-3}\right]$, and $k=k(u)$ is the thermal conductivity $\left[\mathrm{W} \mathrm{m}^{-1}{ }^{\circ} \mathrm{C}^{-1}\right]$.

${ }^{1}$ Thermosorb is a trade name used by Frisby Technologies of Clemmons, NC.

${ }^{2}$ Thinsulate is a trademark of the $3 \mathrm{M}$ Corporation. 
The thermal conductivity of a material $k(u)$ is a constant if the material remains in a single phase during heat transfer. When temperature reaches to the fusion point, $u_{f}$, of the material, the phase of the material changes and the value of $k(u)$ also changes. We let $k_{s}$ and $k_{l}$ be the constant thermal conductivities of the material in solid and liquid phases, respectively.

The enthalpy $h(u)$ is essentially the heat content per unit volume. Defining the enthalpy relative to $0^{\circ} \mathrm{C}, h(u)$ represents the quantity of heat required to raise a unit volume from $0^{\circ} \mathrm{C}$ to $u$, and is expressed by $h(u)=\rho \int_{0}^{u} c\left(u^{\prime}\right) d u^{\prime}$ where $\rho$ is the density and $c(u)$ is the specific heat. Let $c_{s}$ and $c_{l}$ be constant specific heats in the solid and liquid phases, respectively.

When the PCM changes phase from liquid to solid at the fusion temperature $u_{f}$ (or vice versa), it releases (or absorbs) the latent heat $L\left[\mathrm{~J} \mathrm{~kg}^{-1}\right]$ and results in a sharp fall (or jump) in enthalpy of amount $\rho L$ [3]. The relationship between enthalpy and temperature is shown in Figure 1. Figure 1 shows that the enthalpy is not a function of temperature, as enthalpy can be any value in the range $\left[\rho c_{s} u_{f}, \rho\left(c_{s} u_{f}+L\right)\right]$ at the fusion temperature $u_{f}$. However, any given enthalpy corresponds to only one temperature, therefore, temperature is a function of enthalpy. At the beginning of heat transfer, a PCM is in a single phase state, say in the liquid phase for the case of a diver dry suit. Given the initial temperature profile $\mathbf{u}^{(0)}$ and hence the initial enthalpy profile $\mathbf{h}^{(0)}$, the enthalpy at the next time step $t_{0}+\Delta t$ is then determined using equations (11) and (15) (see Section 3), and the temperature profile at $t_{0}+\Delta t$ is

$$
u= \begin{cases}h /\left(\rho c_{s}\right), & \text { if } h<\rho c_{s} u_{f}, \\ u_{f}, & \text { if } \rho c_{s} u_{f} \leq h<\rho\left(c_{s} u_{f}+L\right), \\ u_{f}+\left(h-\rho\left(c_{s} u_{f}+L\right)\right) /\left(\rho c_{l}\right), & \text { if } h \geq \rho\left(c_{s} u_{f}+L\right) .\end{cases}
$$

To model heat transfer through the thickness of a diver's dry suit, the boundary conditions at the water side and the skin side of the garment need 


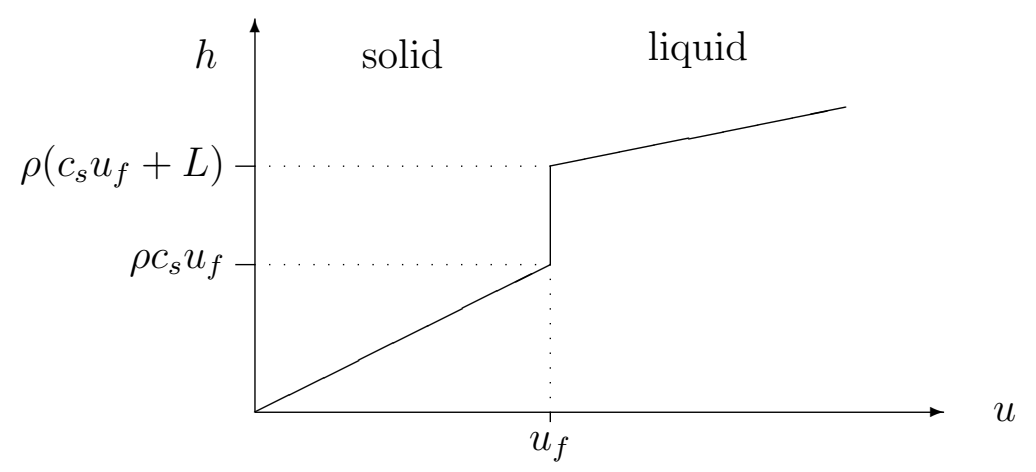

FIgURE 1: The relationship between enthalpy $h$ and temperature $u$.

to be determined. At the water boundary $x=0$, Newton cooling is assumed:

$$
k(u) \frac{\partial u}{\partial x}(0, t)=P\left[u(0, t)-u_{A}\right]
$$

where $P$ is the surface heat transfer coefficient $\left[\mathrm{W} \mathrm{m}^{-2}{ }^{\circ} \mathrm{K}^{-1}\right]$ and $u_{A}$ is the ambient temperature (water temperature).

At the skin-contact boundary $x=l$, since we are interested in determining the rate of heat loss in order to estimate the thermal protection period (how long before the wearer starts to experience a constant heat loss after being exposed to cold water), a constant skin temperature over an extended time period is assumed, hence

$$
u(l, t)=u_{\text {skin }} .
$$

With these boundary conditions, we calculate the heat flux at the skincontact boundary from $t=0$ onwards. When the calculated heat flux approaches a constant, the fabric reaches its steady state of heat transfer. The time used in the transient period is defined as the thermal protection time of the material. Note that the assumed boundary condition is conservative for calculating the thermal protection time. In reality, there is an air gap between the fabric and the diver's skin, which presents a further delay due to the heat conduction between the skin-contact boundary of the fabric and the 
diver's skin. Thus, the definition of the thermal protection period provides a conservative estimate.

If the diver continues to be exposed to the icy water environment after the thermal protection time is exceeded, hypothermia will eventually occur. The time from the fabric approaching to the steady state to the commencement of hypothermia depends upon factors related mainly to the physical condition of the diver. Once hypothermia starts, the boundary condition of the fabric at the skin-contact side will no longer be a constant. The heat transfer will become an unsteady process depending on the rate of the decline of the diver's body temperature. This unsteady heat transfer process is not within the scope of the present study.

\section{The Galerkin finite element discretization}

We employ the first order Euler's scheme for time stepping to re-write equation (1):

$$
\frac{h^{(n+1)}(u)-h^{(n)}(u)}{\Delta t}=\frac{\partial}{\partial x}\left(k(u) \frac{\partial u^{(n)}}{\partial x}\right),
$$

with the initial condition $u(x, 0)=u^{(0)}(x)=u_{\text {skin }}$ and the time step $\Delta t=$ $t_{n+1}-t_{n}$. To ensure the stability of the solution using the explicit forward difference scheme, a restriction of $\max \left\{\frac{\Delta t}{(\Delta x)^{2}} \frac{k_{s}}{\rho c_{s}}, \frac{\Delta t}{(\Delta x)^{2}} \frac{k_{l}}{\rho c_{l}}\right\}<\frac{1}{2}$ is applied on the time step $\Delta t[2]$.

We then apply the weighted residual method [9] to equation (5) and integrate by parts the second derivative term in the resulting equation to obtain

$$
\int_{l} H^{(n+1)}(u) W_{i}(x) d x=\left[k(u) \frac{\partial u^{(n)}}{\partial x} W_{i}(x)\right]_{x=0}^{x=l}-\int_{l} k(u) \frac{\partial u^{(n)}}{\partial x} \frac{\partial W_{i}(x)}{\partial x} d x
$$


where

$$
H^{(n+1)}(u)=\frac{h^{(n+1)}(u)-h^{(n)}(u)}{\Delta t}
$$

is the rate of change of the enthalpy; $l$ is the thickness of the garment and $W_{i}(i=1,2, \ldots)$ are the arbitrary weighting functions equal in number to the number of equations.

We now impose the essential boundary condition, equation (4), on $x=l$ and use the natural boundary condition, equation (3), in equation (6) to give

$$
\begin{aligned}
& \int_{l} H^{(n+1)}(u) W_{i}(x) d x \\
&=-\int_{l} k(u) \frac{\partial u^{(n)}}{\partial x} \frac{\partial W_{i}(x)}{\partial x} d x-P\left[u^{(n)}(0) W_{i}(0)-u_{A} W_{i}(0)\right] .
\end{aligned}
$$

The region $0 \leq x \leq l$ is discretized into $N$ three node $1 \mathrm{D}$ elements of equal length $\Delta x=l / N$, giving a total of $2 N+1$ nodes. The finite element process seeks the solution of the unknown function $\mathbf{u}(x, t)$ in the approximate form

$$
u^{(n)}(x) \approx \sum_{j=1}^{2 N+1} N_{j}(x) u_{j}^{(n)},
$$

where $u_{j}^{(n)}$ are the nodal values of the temperature at the $n$th time step. $N_{j}(x)$ are prescribed piecewise quadratic shape functions and $N_{j}\left(x_{i}\right)=1$ for $i=j$ and $N_{j}\left(x_{i}\right)=0$ for $i \neq j$.

We approximate the unknown function $H^{(n+1)}(u)$ in a similar fashion:

$$
H^{(n+1)}(u) \approx \sum_{j=1}^{2 N+1} N_{j}(x) H_{j}^{(n+1)},
$$

where $H_{j}^{(n+1)}$ are the nodal values of the enthalpy rate $H(u, t)$ at the $(n+1)$ th time step. 


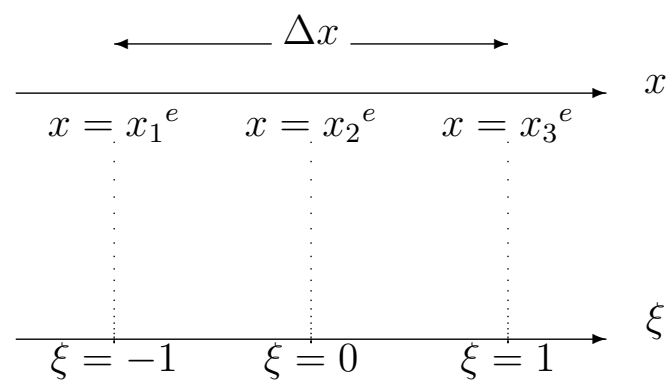

FiguRE 2: Illustration of the local coordinate system $\xi$ and the global coordinate system $x$ on a general element $e$.

In the Galerkin finite element method, the weighting functions take the form of the shape functions

$$
W_{i}=N_{i}, \quad i=1,2, \ldots, 2 N+1 .
$$

Substituting equations (8), (9) and (10) into equation (7) yields the set of discretized equations

$$
E_{i, j} H_{j}^{(n+1)}=K_{i, j} u_{j}^{(n)}+f_{j}, \quad i, j=1,2, \ldots, 2 N+1,
$$

where

$$
\begin{gathered}
K_{i, j}=-\int_{l} k(u) \frac{\partial N_{i}(x)}{\partial x} \frac{\partial N_{j}(x)}{\partial x} d x-P N_{i}(0) N_{j}(0), \\
f_{j}=P u_{A} N_{j}(0) \quad \text { and } \quad E_{i, j}=\int_{l} N_{i}(x) N_{j}(x) d x .
\end{gathered}
$$

For effective implementation of the finite element scheme, we use a local coordinate system $\xi$ on a general element $e$ (the element number). The origin of the local coordinate system $\xi$ coincides with the centre of the element, $e$, as shown in Figure 2. The relationship between the global and local coordinate 
systems is $\xi=\frac{2}{\Delta x}\left(x-x_{2}{ }^{e}\right)$ and $\frac{\partial x}{\partial \xi}=\frac{\Delta x}{2}$. The quadratic shape functions defined by the local coordinate system $\xi$ are

$$
N_{1}(\xi)=\frac{\xi(\xi-1)}{2}, \quad N_{2}(\xi)=1-\xi^{2}, \quad N_{3}(\xi)=\frac{\xi(\xi+1)}{2} .
$$

Equation (11) is now written over the domain of a general element $e$ as

$$
E_{i, j}^{e} H_{j}^{(n+1)}=K_{i, j}^{e} u_{j}^{(n)}+f_{j}^{e}, \quad i, j=1,2,3,
$$

where

$$
\begin{aligned}
K_{i, j}^{e} & = \begin{cases}-\frac{2}{\Delta x} \int_{-1}^{1} k(u) \frac{\partial N_{i}(\xi)}{\partial \xi} \frac{\partial N_{j}(\xi)}{\partial \xi} d \xi-P N_{i}(-1) N_{j}(-1), & e=1, \\
-\frac{2}{\Delta x} \int_{-1}^{1} k(u) \frac{\partial N_{i}(\xi)}{\partial \xi} \frac{\partial N_{j}(\xi)}{\partial \xi} d \xi, & e \neq 1,\end{cases} \\
f_{j}^{e} & =\left\{\begin{array}{ll}
P u_{A} N_{j}(-1), & e=1, \\
\overrightarrow{0}, & e \neq 1,
\end{array} \text { and } E_{i, j}^{e}=\frac{\Delta x}{2} \int_{-1}^{1} N_{i}(\xi) N_{j}(\xi) d \xi .\right.
\end{aligned}
$$

When the element $e$ is in liquid phase, we substitute $k(u)=k_{l}$ in equation (12), and similarly substitute $k(u)=k_{s}$ for solid phase. When the element is in the phase change state,

$$
\begin{aligned}
K_{i, j}^{e}=-\frac{2}{\Delta x} \int_{-1}^{0}\left[k_{s}+\left(k_{l}-k_{s}\right)(\xi+1)\right] & \frac{\partial N_{i}(\xi)}{\partial \xi} \frac{\partial N_{j}(\xi)}{\partial \xi} d \xi \\
& -\frac{2 k_{l}}{\Delta x} \int_{0}^{1} \frac{\partial N_{i}(\xi)}{\partial \xi} \frac{\partial N_{j}(\xi)}{\partial \xi} d \xi,
\end{aligned}
$$

if the change is in the first half of the element. Otherwise

$$
\begin{aligned}
K_{i, j}^{e}=-\frac{2 k_{l}}{\Delta x} \int_{-1}^{0} \frac{\partial N_{i}(\xi)}{\partial \xi} & \frac{\partial N_{j}(\xi)}{\partial \xi} d \xi \\
& -\frac{2}{\Delta x} \int_{0}^{1}\left[k_{s}+\left(k_{l}-k_{s}\right) \xi\right] \frac{\partial N_{i}(\xi)}{\partial \xi} \frac{\partial N_{j}(\xi)}{\partial \xi} d \xi
\end{aligned}
$$


The modifications given by equations (13) and (14) ensure the correct physical modeling of the phase change.

Using the local coordinate system, we only need to calculate the local matrices $K_{i, j}^{e}$ (for each phase) and $E_{i, j}^{e}$ and the local vector $f_{j}^{e}$ once, then assemble all the elements to form the global matrices $K_{i, j}$ and $E_{i, j}$ and the global vector $f_{j}$ and hence solve equation (11) for $H_{j}^{(n+1)}$. The enthalpy at $t+\Delta t$ is

$$
h_{j}^{(n+1)}=\Delta t H_{j}^{(n+1)}+h_{j}^{(n)} .
$$

Subsequently the temperature profile $\mathbf{u}^{(n+1)}$ is determined using equation (2).

\section{Results and discussions}

The finite element algorithm in Section 3 was coded in Maple to investigate the thermal performance of a diver's dry suit using four different garments made from two materials, Thinsulate and the PCM encapsulated material in Thermosorb (as used by Gear et al. [2]). The material properties are given in Table 1. The four types of garments considered here are

1. 1-layer Thinsulate $(100 \% \mathrm{~T})$;

2. 1-layer PCM (100\% PCM);

3. 2-layer composite (40\% T - 60\% PCM); and

4. 4-layer composite (20\% $\mathrm{T}-30 \% \mathrm{PCM}-20 \% \mathrm{~T}-30 \% \mathrm{PCM})$.

All the garments have a constant thickness $0.0127 \mathrm{~m}$, which is discretized into 10 elements with a total of 21 nodal points. The initial temperature of the garments is $32^{\circ} \mathrm{C}$. It is reasonable to assume that the diver's skin temperature be constant at $32^{\circ} \mathrm{C}$. Typical values of surface heat transfer coefficient, for 


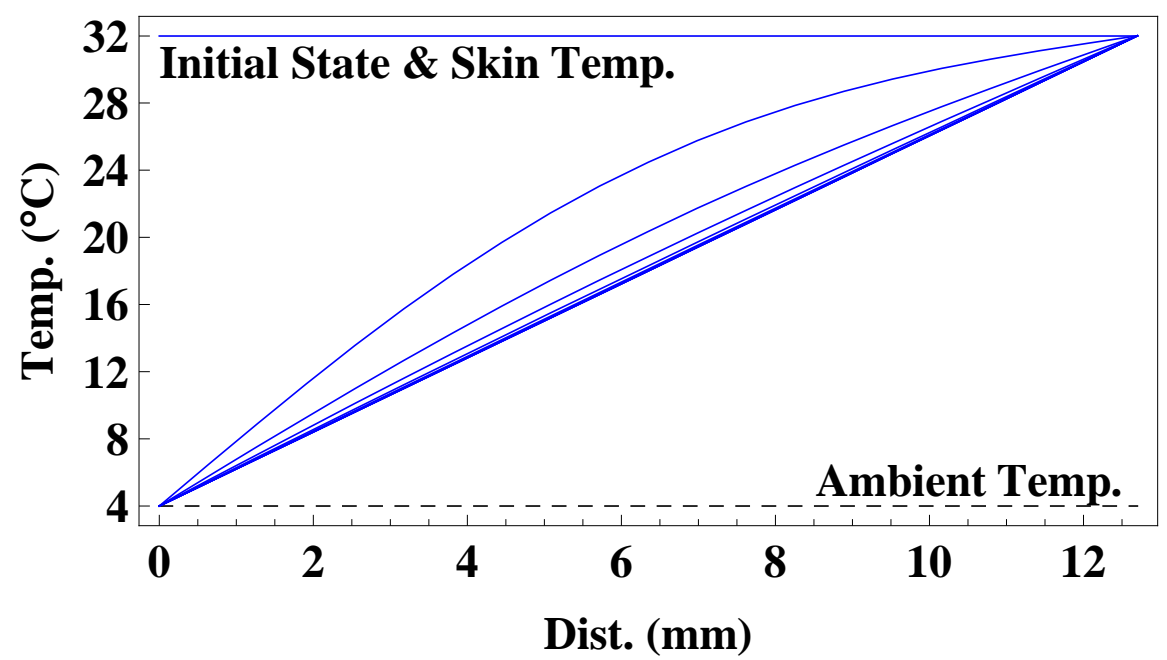

Figure 3: Temperature profiles plotted at 0.5 minute intervals for 30 minutes for 1-layer Thinsulate.

icy water, range from 100 to 1000 [2]. A value of $P=600 \mathrm{~W} \mathrm{~m}^{-2}{ }^{\circ} \mathrm{C}^{-1}$ is used in the present investigation. The PCM is initially all liquid.

Figure 3 shows the temperature profiles of 1-layer Thinsulate garment at 0.5 minute intervals for 30 minutes after the diver and suit are immersed in water of $4^{\circ} \mathrm{C}$. The horizontal axis represents the garment thickness and the vertical axis represents the temperature distribution along the thickness of the garment. It shows clearly that the temperature profile drops rapidly and approaches the steady state within the first 2 minutes. Figure 4 shows the temperature profile for the PCM only garment. There is considerable longer thermal protection for the 1-layer PCM garment. Figures 5 and 6 present the temperature profiles of the 2-layer composite and 4-layer composite garments at the same time interval and over the same time period, respectively. Comparing with Figures 3 and 4, the rate of the temperature drop of the composites is slightly higher than the 1-layer PCM; however, it is significantly lower than the 1-layer Thinsulate. The results shown in Figures 3 


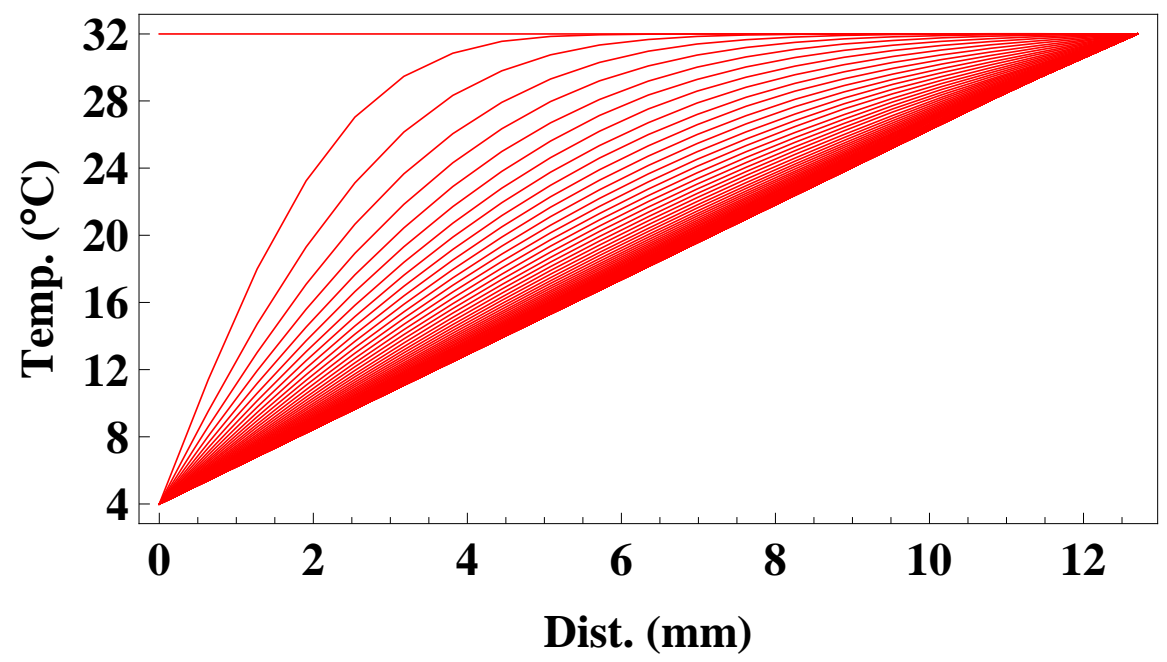

Figure 4: Temperature profiles plotted at 0.5 minute intervals for 30 minutes for 1-layer PCM.

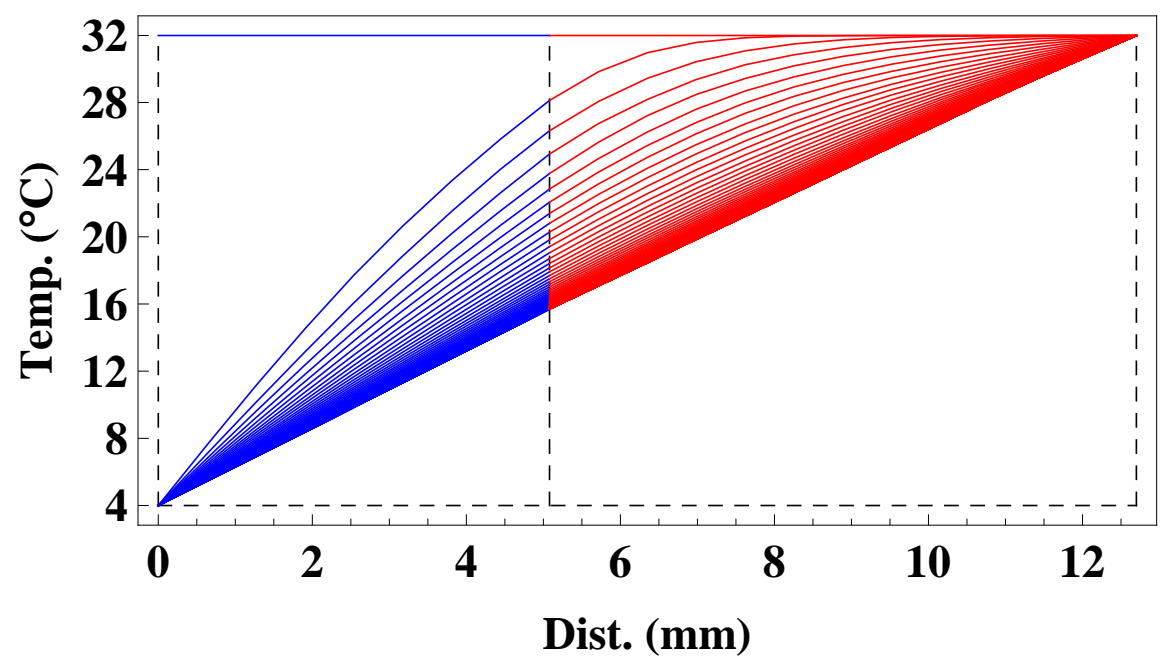

Figure 5: Temperature profiles plotted at 0.5 minute intervals for 30 minutes for the 2-layer composite. 


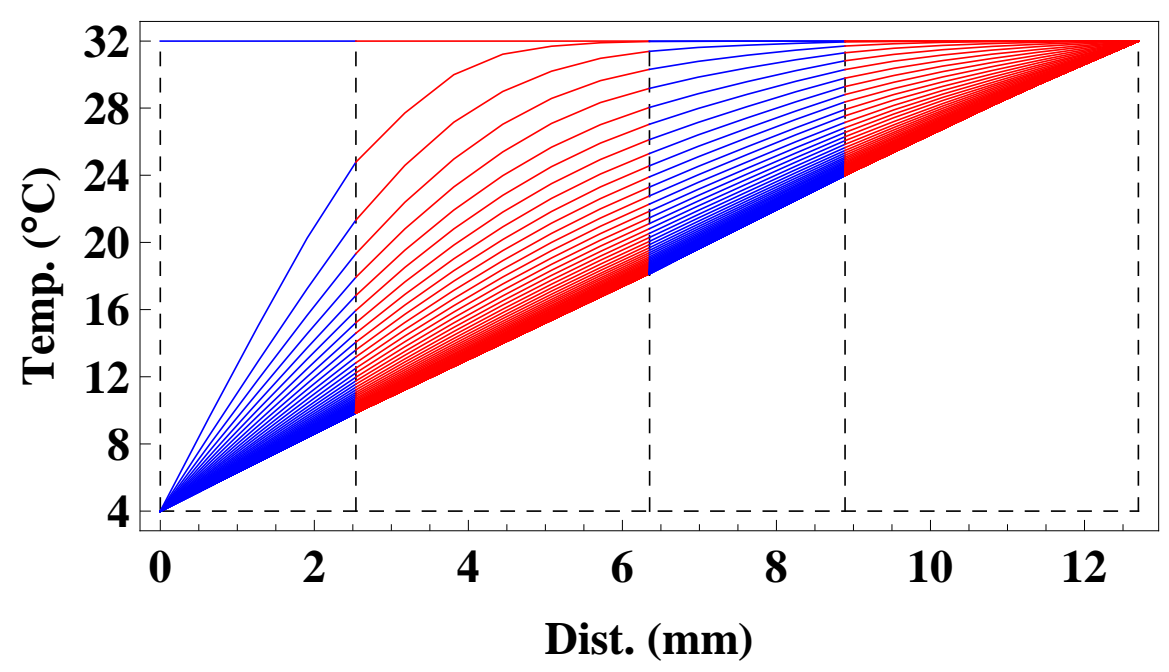

Figure 6: Temperature profiles plotted at 0.5 minute intervals for 30 minutes for the 4-layer composite.

TABLE 1: Thermal properties of Thinsulate and PCM [2].

\begin{tabular}{|c|c|c|c|}
\hline & PCM solid & PCM liquid & Thinsulate \\
\hline Specific heat $\left[\mathrm{J} \mathrm{kg}^{-1}{ }^{\circ} \mathrm{K}^{-1}\right]$ & 1483 & 1620 & 1129.68 \\
Therm. cond. $\left[\mathrm{W} \mathrm{m}^{-1}{ }^{\circ} \mathrm{K}^{-1}\right]$ & 0.03478 & 0.03798 & 0.03286 \\
\cline { 2 - 2 } Density $\left[\mathrm{kg} \mathrm{m}^{-3}\right]$ & \multicolumn{2}{|c|}{121.43} & 52.86 \\
Latent heat $\left[\mathrm{J} \mathrm{kg}^{-1}\right]$ & \multicolumn{2}{|c|}{99424.87} & \\
Fusion Temp. ${ }^{\circ} \mathrm{C}$ & \multicolumn{2}{|c|}{28.3333} & \\
\hline
\end{tabular}


to 6 indicate that the 1-layer PCM performs the best. However, in reality PCM alone cannot be directly used in clothing. It has to be encapsulated into microscopic or macroscopic balls and packed between layers of fabrics in the form of a composite. The 1-layer PCM garment analysis presented here is for the purpose of comparison to the Thinsulate and composites, and also provides the upper limit of the numerical results.

Figure 7 gives a comparison of the heat fluxes at the skin-contact boundary against time between the four garments. It shows that the heat flux of the 1-layer Thinsulate approaches to a constant value within the first 3 minutes, while 1-layer PCM takes about 30 minutes. For the two composites, the times for their heat flux at the skin-contact boundary approaching to a constant value are almost the same and at about 26 minutes. However, within the first 12 minutes, the heat flux value at the skin-contact boundary of the 4-layer composite is less than the 2-layer composite at any a given time. This means the 4-layer composite protects the wearer better since the heat loss is less with the 4-layer composite suit than the 2-layer composite suit. A summary of the results is given in Table 2, in which the time to reach the steady state is defined as the time when the increase of the heat flux is less than $10^{-2} \mathrm{~W} \mathrm{~m}^{-2} \mathrm{~min}^{-1}$. These results are very close to the results reported by Gear et al. [2]. The location of the moving boundary (the phase change position), agrees with both the finite difference method (FDM) of Gear et al. [2] and the results of Nuckols [8]. The difference between the FDM and FEM results is that the FDM result (of 100 grid points) produces a stepped curve in heat flux plots for garments containing PCM; however, the step distance could be reduced by half each time the grid is doubled; whereas the FEM results give smooth heat flux curves though only a total of 21 nodes are used. Regarding the computational accuracy, if linear shape functions were used, the system of algebraic equations obtained by FEM would be the same as that by FDM. Since the present FEM algorithm uses quadratic shape functions, the accuracy of the FEM results is higher than that of the FDM results. 
TABLE 2: Comparison of time, heat flux and phase change position at steady state obtained from Finite Difference and Finite Element Methods.

\begin{tabular}{|l|ccc|}
\hline Garment types & \multicolumn{3}{|c|}{ Finite Difference Method } \\
& $\begin{array}{c}\text { Time } \\
\text { (mins) }\end{array}$ & $\begin{array}{c}\text { Heat flux } \\
\left(\mathrm{W} \mathrm{m}^{-2}\right)\end{array}$ & $\begin{array}{c}\text { Phase change } \\
\text { position }(\mathrm{mm})\end{array}$ \\
\hline 1-layer Thinsulate & 3 & 72.136 & $\mathrm{NA}$ \\
1-layer PCM & 30 & 77.252 & 10.897 \\
2-layer Thin./PCM $(40 \%-60 \%)$ & 25 & 75.495 & 10.855 \\
\hline \hline Garment types & \multicolumn{3}{|c}{ Finite Element Method } \\
& Time & Heat flux & Phase change \\
& $($ mins $)$ & $\left(\mathrm{W} \mathrm{m}^{-2}\right)$ & position (mm) \\
\hline 1-layer Thinsulate & 2.5 & 72.447 & NA \\
1-layer PCM & 30 & 77.502 & 10.900 \\
2-layer Thin./PCM $(40 \%-60 \%)$ & 26 & 75.714 & 10.869 \\
\hline
\end{tabular}

\section{Conclusions}

1. The 1-layer PCM material gives the longest thermal protection up to approximately 30 minutes, which is more than ten times longer than the conventional 1-layer Thinsulate fabric;

2. Compared with the 1-layer Thinsulate, the Thinsulate-PCM composites have significantly improved thermal protection, up to 26 minutes; however, doubling the composite layering does not seem to enhance the protection further.

3. The FEM results (using the modified scheme) agree well with the FDM results. The FEM results also show improved accuracy with much fewer nodes.

4. Beyond the thermal protection time, the value of the heat flux at the 


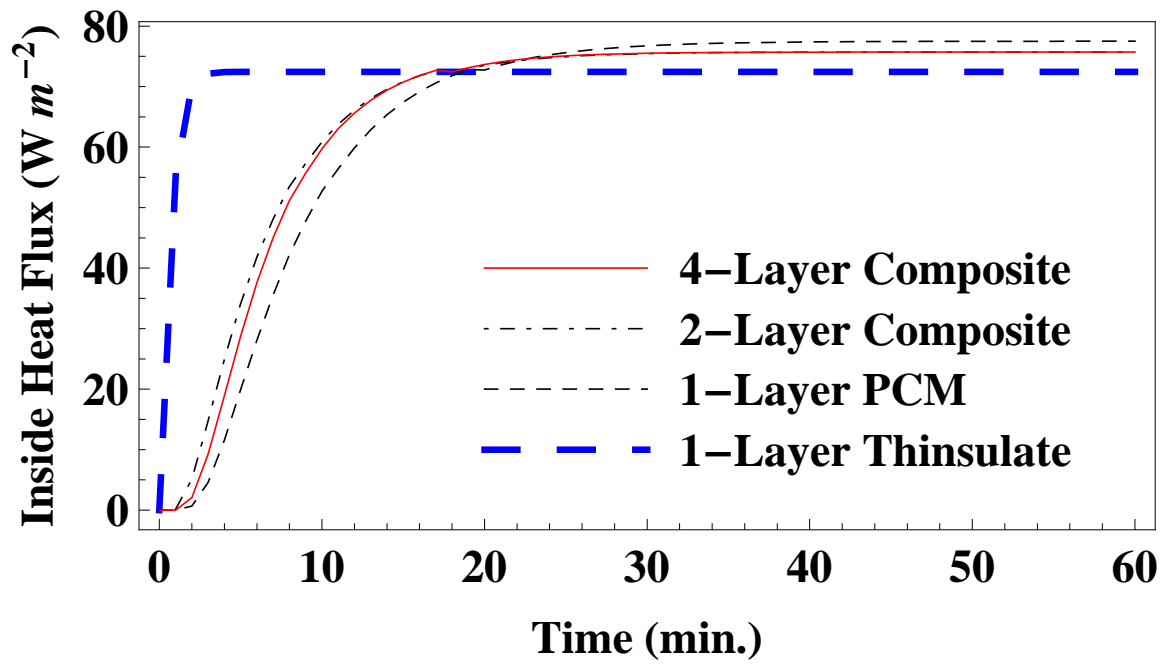

FiguRE 7: Heat flux at the skin boundary against time for 4-layer and 2-layer composites, 1-layer PCM and 1-layer Thinsulate.

skin-contact boundary is the lowest for the 1-layer Thinsulate and highest for the 1-layer PCM. Hence for an extended period, Thinsulate-only fabric would be optimal as its rate of heat loss (measured by the heat flux) is the least beyond its transient heat transfer state.

\section{References}

[1] D. P. Colvin and Y. G. Bryant. Protective clothing containing encapsulated phase change materials. Advances in Heat and Mass Transfer in Biotechnology, HD-Vol. 362/BED-Vol. 40:123-132,1998. C440

[2] J. A. Gear, M. J. Lachut and Y. Ding. Enchanced thermal performance of garments embeded with encapsulated phase change material. 
ANZIAM J., 47(EMAC2005):C137-C151, 2006.

http://anziamj .austms.org.au/V47EMAC2005/Gear C441, C444, C448, C449, C451, C452

[3] J. M. Hill and J. N. Dewynne. Heat Conduction. Applied mathematics and engineering science texts. Blackwell Scientific Publications, 1987. C441, C442

[4] D. C. Hittle and T. L. Andre. A new test instrument and procedure for evaluation of fabrics containing phase-change material. ASHRAE Transactions, 4509:175-182, 2002. C440

[5] G. E. R. Lamb and K. Duffy-Morris. Heat loss through fabrics under ventilation with and without a phase transition additive. Textile Research Journal, pages 261-265, ISSN:0040-5175. C440

[6] G. A. Lane. Solar heat storage: latent heat material. Vol. I, CRC Press, Boco Raton, Florida, 1983. C440

[7] G. N. Mercer, and H. S. Sidhu. Mathematical modelling of the effect of fire exposure on a new type of protective clothing. To appear in ANZIAM J., V49. Proceedings of the 8th Biennial Engineering Mathematics and Applications Conference, EMAC-2007. Editors G. N. Mercer and A. J. Roberts. C440

[8] M. L. Nuckols. Analytical modeling of a diver dry suit enhanced with micro-encapsulated phase change materials. Ocean Engineering, 26:547-564, 1999. doi:10.1016/S0029-8018(98)00001-8 C440, C441, C452

[9] O. C. Zienkiewicz, R. L. Taylor, and J. Z. Zhu. The finite element method: its basis and fundamentals, sixth edition, Elsevier Butterworth-Heinemann 2005. ISBN 0750663200 C444 


\section{Author addresses}

1. Y. Ding, School of Mathematical and Geospatial Sciences, RMIT University, Melbourne, Australia. mailto:yan.ding@rmit.edu.au

2. J. A. Gear, School of Mathematical and Geospatial Sciences, RMIT University, Melbourne, Australia.

3. K. N. Tran, School of Mathematical and Geospatial Sciences, RMIT University, Melbourne, Australia. 